\title{
Development and implementation of an Integrated Water Resources Management System (IWRMS)
}

\author{
W.-A. Flügel ${ }^{1}$ and C. Busch ${ }^{2}$ \\ ${ }^{1}$ Institute for Geography, University of Jena, Germany \\ ${ }^{2}$ Codematix GmbH, Jena, Germany
}

\begin{abstract}
One of the innovative objectives in the EC project BRAHMATWINN was the development of a stakeholder oriented Integrated Water Resources Management System (IWRMS). The toolset integrates the findings of the project and presents it in a user friendly way for decision support in sustainable integrated water resources management (IWRM) in river basins. IWRMS is a framework, which integrates different types of basin information and which supports the development of IWRM options for climate change mitigation. It is based on the River Basin Information System (RBIS) data models and delivers a graphical user interface for stakeholders. A special interface was developed for the integration of the enhanced DANUBIA model input and the NetSyMod model with its Mulino decision support system (mulino mDss) component. The web based IWRMS contains and combines different types of data and methods to provide river basin data and information for decision support. IWRMS is based on a three tier software framework which uses (i) html/javascript at the client tier, (ii) PHP programming language to realize the application tier, and (iii) a postgresql/postgis database tier to manage and storage all data, except the DANUBIA modelling raw data, which are file based and registered in the database tier. All three tiers can reside on one or different computers and are adapted to the local hardware infrastructure. IWRMS as well as RBIS are based on Open Source Software (OSS) components and flexible and time saving access to that database is guaranteed by web-based interfaces for data visualization and retrieval. The IWRMS is accessible via the BRAHMATWINN homepage: http://www. brahmatwinn.uni-jena.de and a user manual for the RBIS is available for download as well.
\end{abstract}

\section{Introduction and objectives}

Integrated river basin studies as done in the BRAHMATWINN project require a common data and information platform that was provided by the River Basin Information System (RBIS) developed by at the FSU-Jena (Flügel, 2007, 2009; Kralisch et al., 2009). The IWRMS builds on this well tested toolset and enhanced the latter by integrating data and methods from the deliverables of the BRAHMATWINN project, like the time series of station data, the results of the DANUBIA modelling system (Mauser and Bach, 2009) and the analysis done by means of the NetSyMod-mulino decision support system (mDss) (Giupponi et al., 2008).

The overall objective of the IWRMS development was to offer users and decision makers the DANUBIA modelling results (Chapter 7) together with the "what-if?" scenarios elaborated by the mDss (Chapter 8 ). In addition analysis tools should enable users to support decision making for adap-

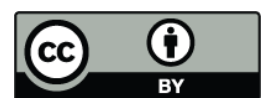

Correspondence to: W.-A. Flügel

(c5wafl@uni-jena.de) tive IWRM option design accounting for impacts of climate change on a basin scale. The overall objective was realised by elaborating on the following scientific-technological objectives:

1. Integration of all BRAHMATWINN research work results, data time series, GIS maps and documents from in the Upper Danube River Basin (UDRB) and the Upper Brahmaputra River Basin (UBRB) respectively into the IWRMS in such a way that they can be used by means of a web-based graphical user interface (GUI).

2. Making the comprehensive system assessment, modelling studies, spatial analysis of water balance components and the integrated socio-economic analysis and scenario evaluation available to local actors, decision makers and planners.

3. Provide additional GUI tools for analysing the information supplied by (i) and (ii) in the twinning basins for IWRM related decision support. 
The challenges related to these objectives were met by building the IWRMS development on the sophisticated RBIS data model structure and designing the system as an easy to use application that extends the RBIS modular framework and deploys all basin specific data accounting for the local computer network structure.

\section{Role within the integrated project}

The RBIS as well as the IWRMS have a central role within the BRAHMATWINN project. RBIS provides the common data and information base accessible via the web to all project partners and authorized stakeholders, and the IWRMS in turn is making use of the RBIS functionalities for decision support. In addition IWRMS provides access to the modelling results of the DANUBIA model applied in the twinning UDRB and UBRB and the "what-if?" scenario evaluation done by means of the mDss. IWRMS furthermore offers different modules that elaborate on this information and provide knowledge based decision support.

The IWRMS thereby offers two important services to users and decision makers, as it firstly makes the knowledge elaborated by the project consortium (see Chapter 1 till Chapter 8) available to decision makers, and secondly provides them with sophisticated tools to make use of the knowledge for decision support related to the design of adaptive IWRM strategies to cope with impacts of climate change.

\section{Scientific methods applied}

\subsection{Definition of IWRMS requirements}

IWRMS requirements were defined in close cooperation with users and stakeholders on several workshops in Germany and Asia as follows:

i. New datasets had to be described by metadata according to ISO19115 standards.

ii. Description of locality had to be implemented applying the Open Geospatial Consortium (OGC) prescriptions.

iii. Different data types and their interrelations, i.e. how applications access data has been grouped as follows:

- GIS data layers (raster and vector) i.e. for geology, land use, river network, or vegetation related to model input data.

- Measured station time series data, i.e. discharge or climate provided by partners and stakeholders for the twinning UDRB and UBRB.

- Raster data provided by the climate modellers (Chapter 2) and the DANUBIA model (Chapter 7) comprising:
- regionalized data of Global Circulation Models (GCM) for different scenarios and modelled climate projections with a grid cell size of $50 \times 50 \mathrm{~km}$;

- output data and parameterisations of the different DANUBIA runs, i.e. 15 modelled hydrometeorological parameter and input meteorological data downscaled to a grid cell of $1 \times 1 \mathrm{~km}$ (Marke, 2008).

- Results from the mDss studies, i.e. analysis matrix, evaluation matrix, weights and options for indicators, sensitivity analysis, and decision making.

- "What-if?" scenarios based on the Special Report on Emissions Scenarios (SRES) of the IPCC (IPCC, 2000).

- Indicators selected in different Delphi exercises (Chapter 6).

- Any type of documents, i.e. reports, graphs, pictures, or spread sheets in standard formats.

- Web based access to original (e.g. xls) and derived (e.g. graphs) data.

These user requirements have been realised by respective modules and interfaces than can be added for use on demand. All data are stored in a set of databases and no distinctions are made between alphanumerical, location based and binary data items. The underlying database uses spatial based extensions (PostGIS) according to the Open Geospatial Consortium (OGC) standards.

\subsection{Design of graphical user interfaces (GUIs)}

The overall objective of the IWRMS was realized by developing new modules and GUIs to handle the data and information exchange between the DANUBIA hydrological model, the RBIS and the mDss. The innovative methodology developed for the data exchange between applications via the IWRMS interfaces defined descriptions of

- how applications want to access input and output data in the database, and

- what kind of API methods will be used by applications to access the data.

In the first part XML-based data dictionaries have been designed, which in the second part the API will make use of to read and write data in a failure-proof manner. Integration of the different kinds of data and indicators for the design of "what-if-scenarios" is provided for the evaluation of prognostic system management options during the decision making processes and when developing adaptive IWRM strategies.

The design of the different GUIs for the IWRM user support was discussed with project partners and stakeholders 


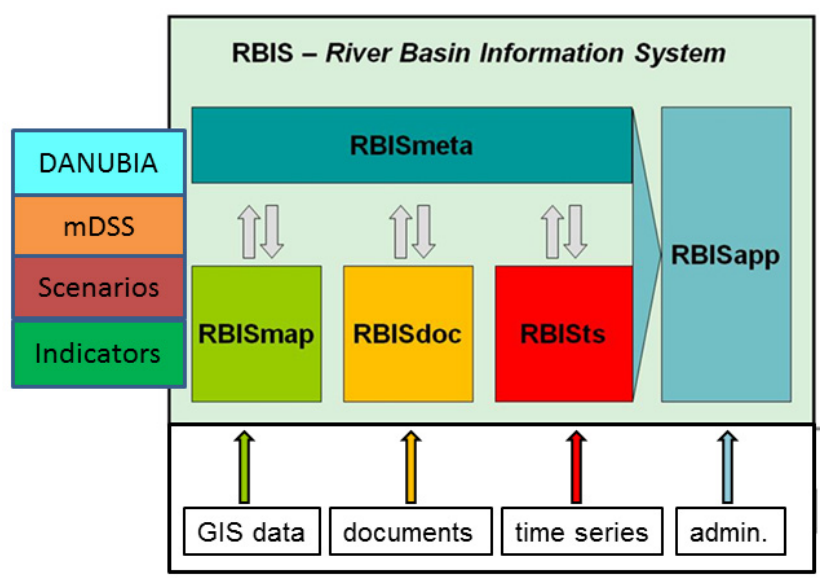

Figure 1. IWRMS data management architecture and data flow.

during IWRMS workshops. It was decided to keep them identical to those of the RBIS in terms of the cascading web map service (WMS) based definitions, and the user management. Hence the GUIs of further IWRMS data, functions and programs like the DANUBIA modelling results, the indicators or the mDss have been designed accordingly. They make use of a similar web based approach and apply a set of adaptable and RBIS compatible extension modules programmed in PHP.

\section{Results achieved and deliverables provided}

\subsection{Enhanced RBIS structure}

The schematically IWRMS model structure is shown in Fig. 1. It shows that the central part of the system is the enhanced RBIS with its four components managing and administrating GIS data, documents, time series and the administration modules. The IWRMS modular components are represented by the interface to the DANUBIA model and the mDss, a module for SRES scenario design and the definition and calculation of indicators to be used for IWRM decision support.

These modules can be selected by the user on demand and thereby reduce the complexity of the system accounting for the level of staff training available at the user side.

Each module of the IWRMS delivers a set of different functions, which can be combined to a new workflow structure. The user rights are administered by the system administrator of the respective stakeholder organization and depending on given user permissions the menu structure is adapted accordingly. This functionality is adjusting the system's complexity to the respective work level of each IWRMS application. A similar fine grained user permission hierarchy rights (hidden, read, download, write, owner) can be assigned to the management of time series data, documents, indicators, GIS layers or GIS maps.

\subsection{Component interaction}

The IWRMS integrates the results of deliverables produced in the BRAHMATWINN project by means of access definitions for semi-automated data interfaces. The latter provides information required by the user, i.e. metadata from file headers, aggregated time series data or coordinate reference systems with minimized controlling user interaction. The IWRMS interface interaction architecture and data fluxes between the IWRMS model components mDss and DANUBIA are shown in Fig. 2 and can be described as follows:

- The mDss needs a tight interface coupling, because data will be exchanged between both systems, especially if indicators from IWRMS are applied in the mDss.

- Opposite the DANUBIA model requires only a lose interface coupling. As the model applies raster input data with grid cell values for each parameter and for each time stamp the latter are linked and registered to the IWRMS only.

- DANUBIA input and output time series data can be extracted by the IWRMS for any given location by selecting the respective raster cell.

\subsection{Enhanced RBIS data management architecture}

Data management and data flow between the different IWRMS components is shown in Fig. 3. They reflect the data models needed to integrate the results generated in the BRAHMATWINN project and can be described as follows:

1. The GIS database management server is a core element of the system providing the different services offered.

2. At the left side modules provide functionalities to manage metadata, time series, raster and vector data.

3. At the right side the component modules provide Desktop GIS, networking (internet/intranet) and indicator management functionalities.

4. Extensive data fluxes between these components are managed by the central server core system which could be located remote or at the user site.

\subsection{Registering new data}

The implementation of the IWRMS modules is based on defined formats and data fluxes and accounts for the data volume and the user requirements towards a web based application. To read a parameter time series output by the DANUBIA model requires the processing of separate files for each time step and is done by means of asynchronous processes as shown in Fig. 4. 


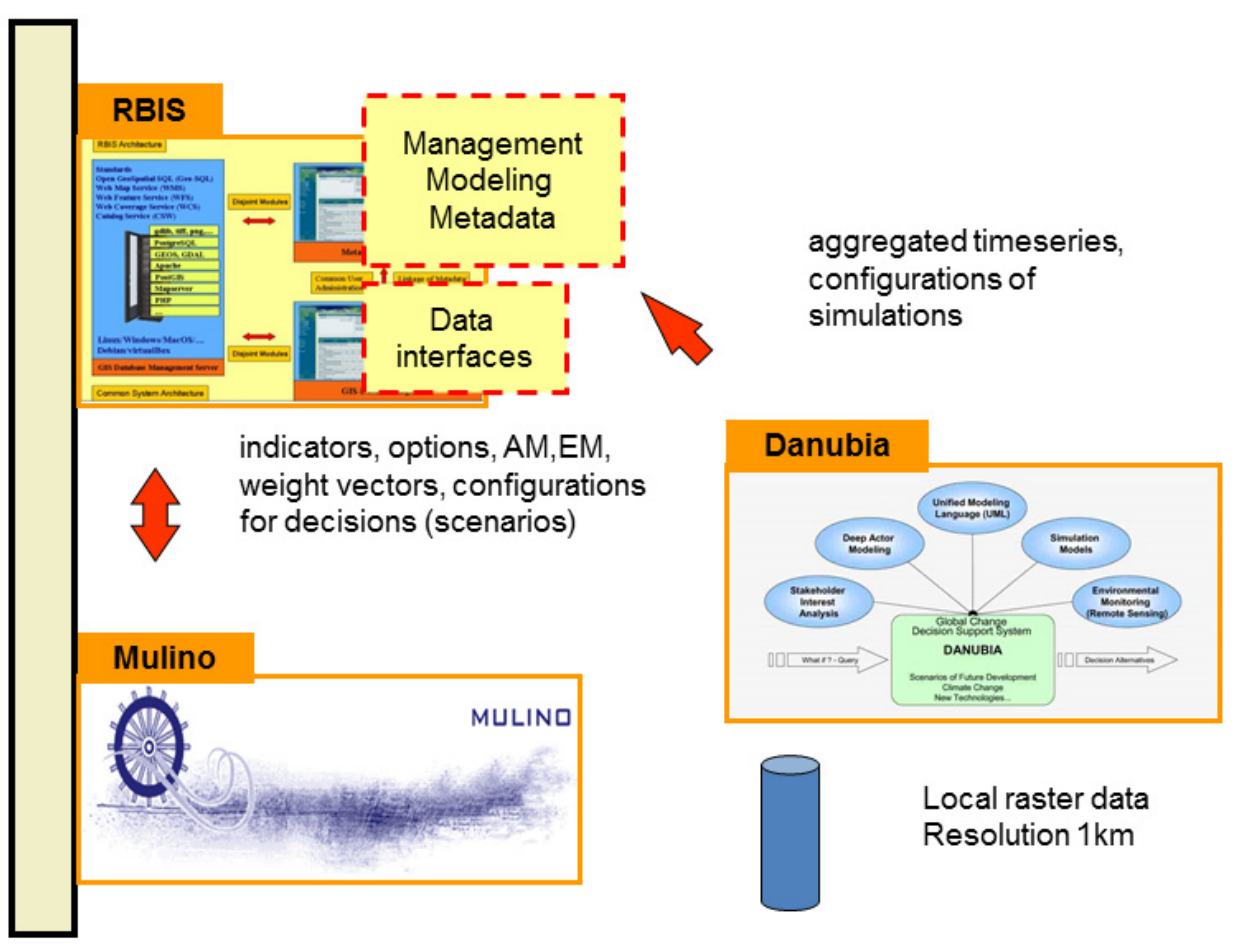

Figure 2. Implemented interaction structure between external applications.

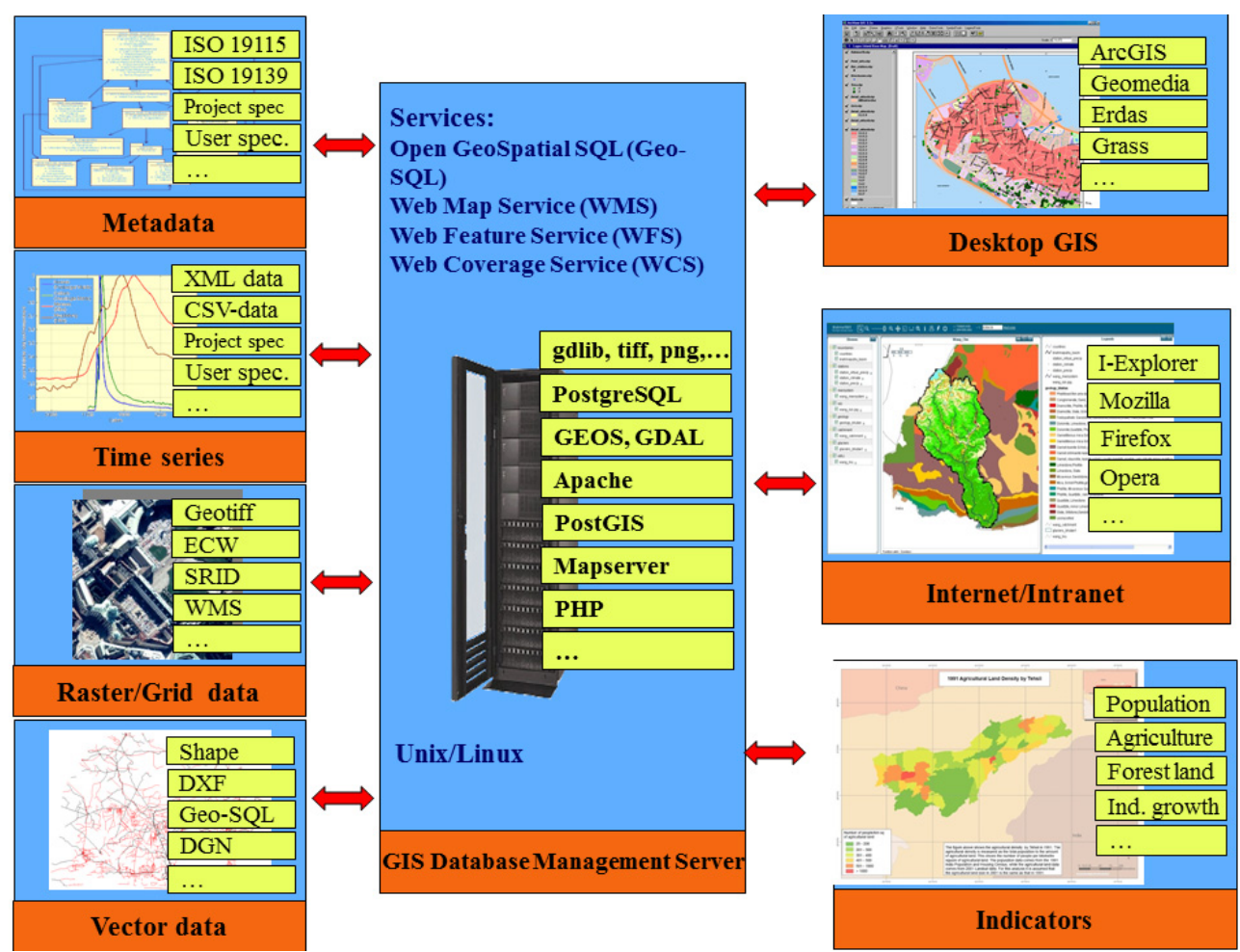

Figure 3. IWRMS data management architecture and data flow. 


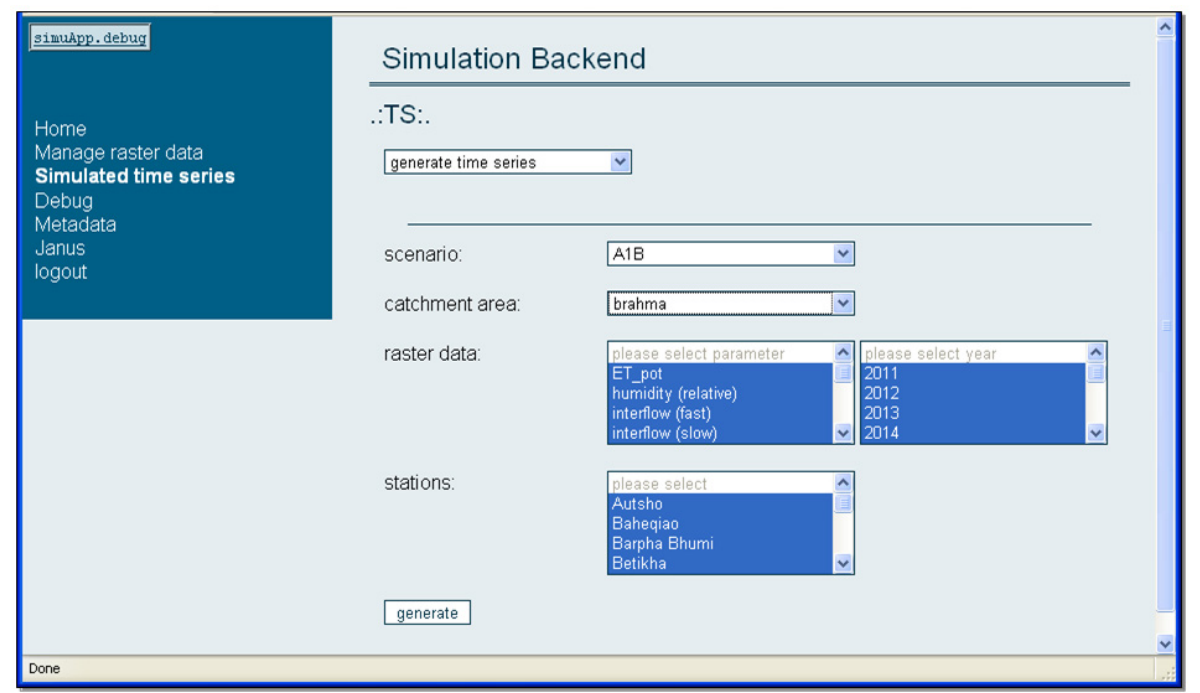

Figure 4. Registering new data to the IWRMS.

The user controlled work flow done by means of GUIs can be described as follows:

1. After registering new data, the first process generates the RBIS and ISO19115 conform metadata associated to the location and its time series.

2. The user in a next step is generating the simulated time series by selecting the parameters specifying time window, location, and name, i.e. precipitation, 2013 and station name.

3. The generated time series data are then extracted from the respective raster file and transposed into a parameter time series that either can be input to RBIS as the IWRMS database or can be downloaded for external use.

4. After loading the extracted time series into the IWRMS they can further be processed by means of functionalities provided by the RBIS.

\subsection{Generating time series for virtual stations}

Virtual stations are represented by grid cells of a river basin model domain. They either can store downscaled meteorological time series or model output data. In the BRAHMATWINN project downscaled data time series have been produced by the climate modelling on a $50 \times 50 \mathrm{~km}$ resolution (Chapter 2) and by the DANUBIA model on a $1 \times 1 \mathrm{~km}$ resolution respectively (Marke, 2008). The DANUBIA model is also using this resolution when writing the model output from historical or projected time periods, thereby producing many terra bytes of data volume.

The IWRMS function developed to extract selected cell data from this huge data volume is completely integrated in the RBISmap application component of RBIS. As shown in Fig. 6 the selection of the raster cell is done on RBISmap by means of a GIS data layer that can address each grid cell of the model domain. After the grid cell has been selected the service applies parts of the workflow described in Sect. 4.4.

This IWRMS service provides a processing method which combines the data from the remote central server with the local catchment server and thereby offers to the user the full information potential produced by the different DANUBIA model runs.

Metadata are added to the generated virtual station and all RBIS data processing functions can be used on the "virtual time series". To avoid an overloading of the central server the number of simultaneously running extraction processes are limited and selection requests exceeding the threshold limit are queued. At present it takes approximately four minutes to generate a complete time series (1970 to 2080, one hour time resolution) with a complete parameter set (20 parameters) for one virtual station.

\subsection{Integration of the mDss system}

The integration of the mDss package was implemented by applying the same modular approach. IWRMS manages different $\mathrm{mDss}$ core program versions, the basin specific configuration files and the associated results of the different Delphi rounds (Nevo and Chan, 2007). IWRMS can either be deployed to represent the results, together with all metadata as relevant information or to run the decision process again locally. Users can change general regulations and can adapt the $\mathrm{mDss}$ configuration to its local needs. Different runs can later be added together with new metadata to IWRMS depending on the permissions given to the user. 


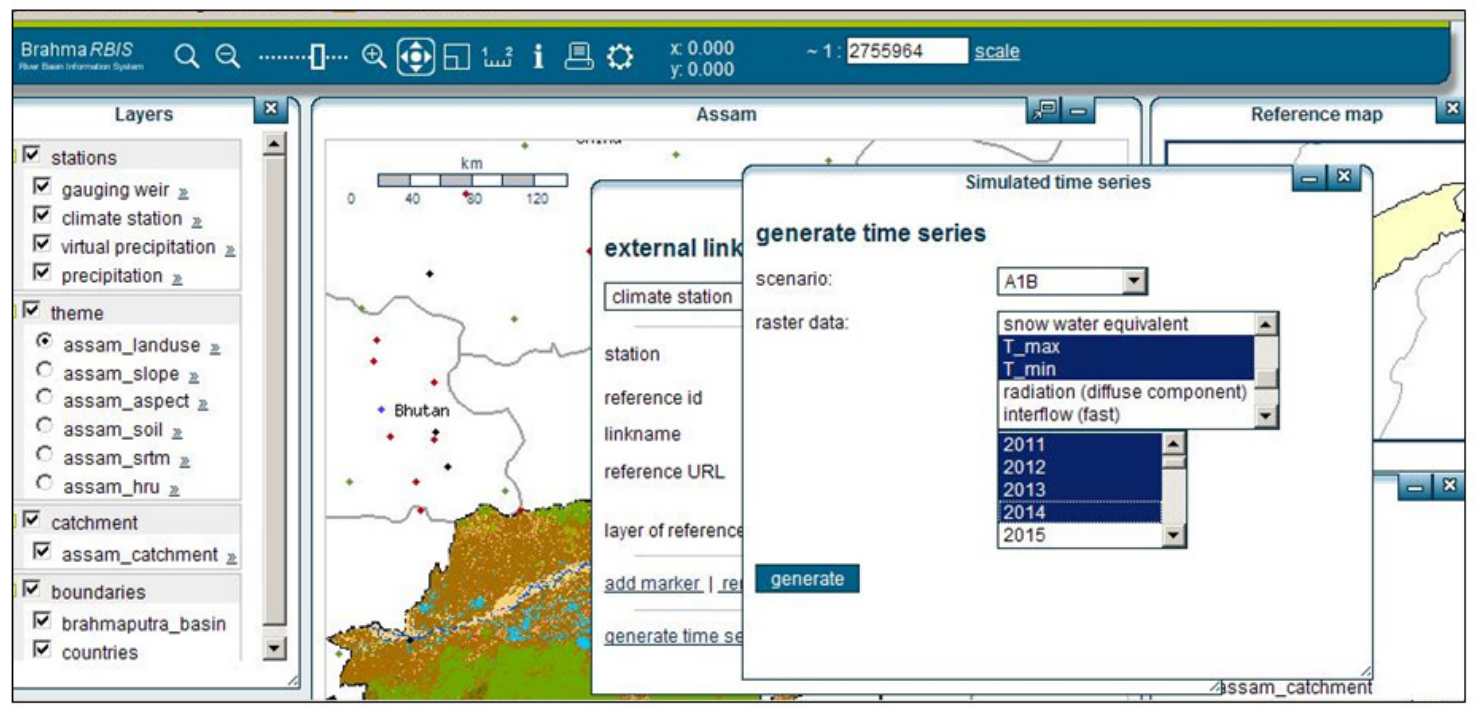

Figure 5. Extracting downscaled data time series from grid cells of a model domain.

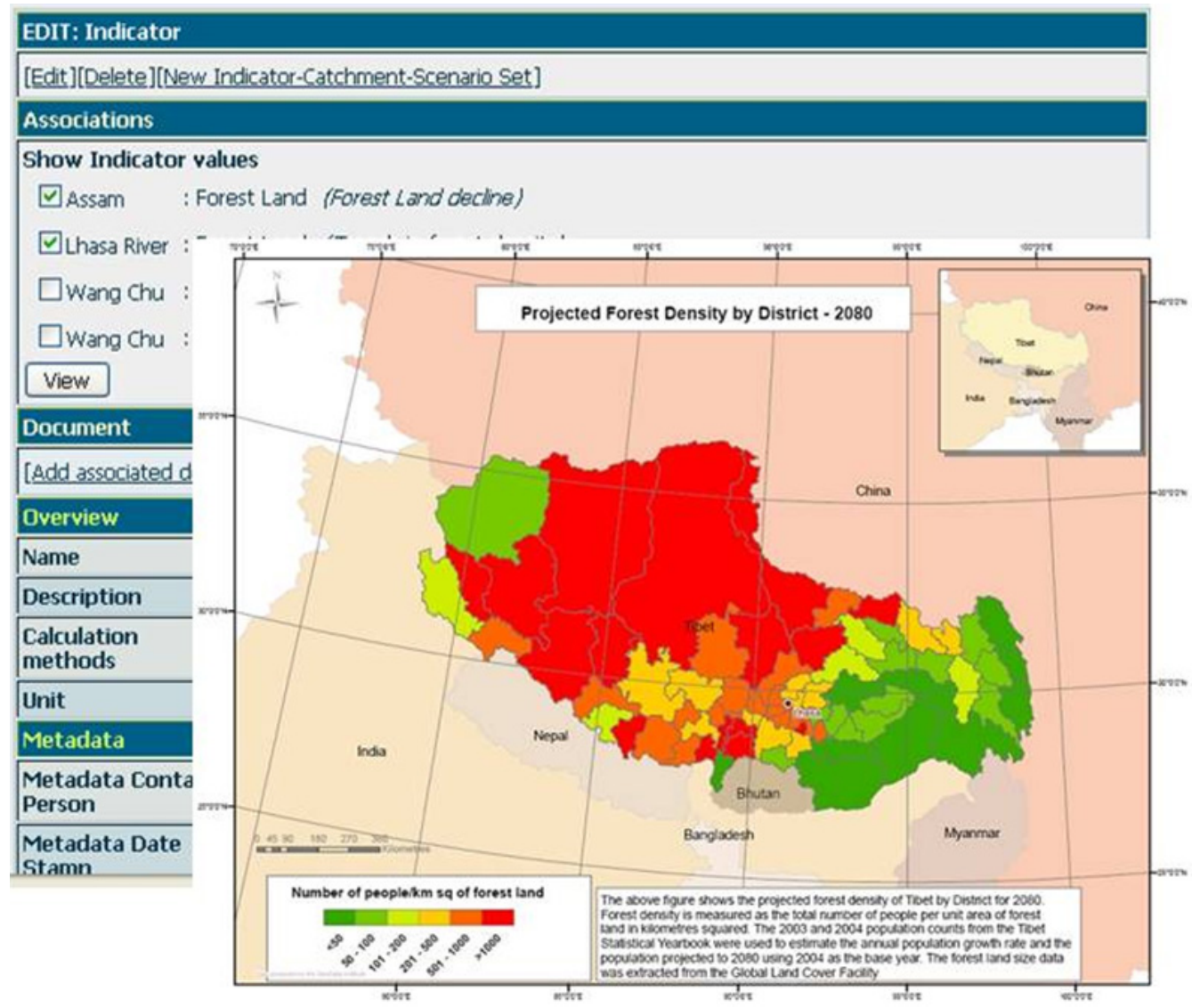

Figure 6. IWRMS components RBISscen and RBISind for indicator management. 

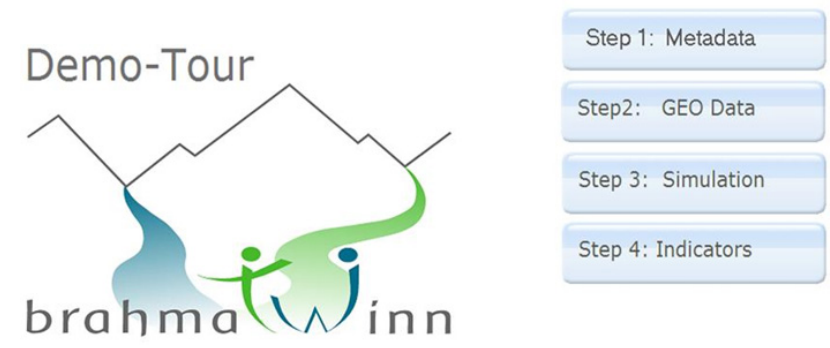

Figure 7. Start GUI for the IWRMS demo-tour of the BRAHMATWINN project.

\subsection{Indicators and IPCC based "what-if?" scenarios}

The use of indicators to analyse scenario based climate and hydrological model projections was realized by developing and implementing the modules RBISscen and RBISind. To meet expressed user needs, the results and dependencies of different indicators can be saved in different user selected formats.

RBISscen delivers an extended database structure and PHP application to integrate the different SRES scenarios (IPCC, 2000) together with additional metadata about the river basin, socio-economic data and the hydrological modelling results.

RBISind integrates indicators of different types as values, normalized values, or documents and assigns them to river basins and user selected "what-if?" scenarios (Chapter 8) by associating measured 'real world' information with the parameters and definitions of the respective "what-if?" scenarios. As shown in Fig. 6 the "what-if?" scenarios were linked to IWRMS metadata, i.e. stations and associated time series or binary documents, thus providing the required knowledge basis for developing and evaluating IWRM options for climate change adaptation.

\subsection{Documentation of the IWRMS toolset}

The system has been documented by means of a user friendly manual and comprehensive online help assistance for each GUI and item. A flash based interactive demo tour tutorial (Fig. 7) describes all functions and steps to work with the system, how to insert new metadata, upload GIS layers, create maps, manage time series, model data registration, virtual time series generation, and indicator management. The guided tour is harmonised with the user manual and can be executed either by a web browser or as a standalone application. The flash tutorial is available via the website http://www.brahmatwinn.uni-jena.de.

\section{Contributions to sustainable IWRM}

The development of the IWRMS presented herein can be seen as an innovative milestone for knowledge based decision support in IWRM. If used together with the many terra bytes of data provided by the BRAHMATWINN project it offers a wide range of decision support for water and land managers, planners and all other kind of decision makers in the UDRB and the UBRB, respectively.

\section{Conclusions and recommendations}

The IWRMS, although presented herein as a "final product" of the BRAHMATWINN project is subject for enhancement and improvement that can be incorporated if applied in other IWRM related research projects. This process has already been started since the end of the project in December 2009.

Lessons learned in the BRAHMATWINN project are that a sophisticated training is a prerequisite to explore the potential of the system. Stakeholder organisations should associate permanent staff with the application of the system and this staff must be trained properly to form the core of further in-house training. If this is provided the system will offer its full potential also for other river basin IWRM challenges that are elaborated by the stakeholder organisation.

Acknowledgements. The cooperation and support from BRAHMATWINN partners and stakeholders was highly appreciated and is acknowledged hereby. Acknowledgement is also given to the EC which funded the IWRMS development in the BRAHMATWINN project under the contract number 036952.

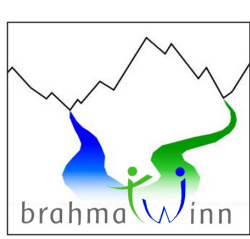

The interdisciplinary BRAHMATWINN EC-project carried out between 2006-2009 by European and Asian research teams in the UDRB and in the UBRB enhanced capacities and supported the implementation of sustainable Integrated Land and Water Resources Management (ILWRM).

\section{References}

Flügel, W.-A.: The Adaptive Integrated Data Information System (AIDIS) for global water research, Water Resources Management (WARM) Journal, 21, 199-210, 2007.

Flügel, W.-A.: Applied Geoinformatics for sustainable IWRM and climate change impact analysis, Technology, Resource Management \& Development, 6, 57-85, 2009.

Giupponi, C., Sgobbi, A., Mysiak, J., Camera, R., and Fassio, A.: NetSyMoD - An Integrated Approach for Water Resources Management, in: Integrated Water Management, edited by: Meire, P., Coenen, M., Lombardo, C., Robba, M., and Sacile, R., Springer, Netherlands, 69-93, 2008.

IPCC, Intergovernmental Panel on Climate Change: Emissions Scenarios. A Special Report of IPCC Working Group III, 27 pp., 2000.

Kralisch, S., Zander, F., and Krause, P.: Coupling the RBIS Environmental Information System and the JAMS Modelling Framework, in: Proc. 18th World IMACS/and MODSIM09 International Congress on Modelling and Simulation, edited by: Anderssen, R., Braddock, R., and Newham, L., Cairns, Australia, 902-908, 2009. 
Marke, T.: Development and Application of a Model Interface to couple Regional Climate Models with Land Surface Models for Climate Change Risk Assessment in the Upper Danube Watershed, Dissertation der FakultätfürGeowissenschaften, DigitaleHochschulschriften der LMU München, 188, available at: http://edoc.ub.uni-muenchen.de/9162/, München, 2008.
Mauser, W. and Bach, H.: PROMET - Large scale distributed hydrological modelling to study the impact of climate change on the water flows of mountain watersheds, J. Hydrol., 376, 362-377, 2009.

Nevo, D. and Chan, Y. E.: A Delphi study of knowledge management systems: Scope and requirements, Inform. Manage., 44, 583-597, 2007. 